\title{
Análise de Rede na Identificação de Habilidades Relacionadas ao Pensamento Computacional
}

\author{
Ana Liz Souto O. Araujo ${ }^{1,2}$, Wilkerson L. Andrade ${ }^{1}$, Dalton D. S. Guerrero ${ }^{1}$, \\ Monilly Ramos A. Melo ${ }^{3}$, Isabelle Maria Lima de Souza ${ }^{1}$ \\ ${ }^{1}$ Laboratório de Práticas de Software (SPLab) \\ Universidade Federal de Campina Grande (UFCG) \\ ${ }^{2}$ Departamento de Ciências Exatas (DCX) \\ Universidade Federal da Paraíba (UFPB) \\ ${ }^{3}$ Laboratório de Neuropsicologia e Inovação Tecnológica \\ Universidade Federal de Campina Grande (UFCG) \\ analiz@dcx.ufpb.br, \{wilkerson, dalton\}@computacao.ufcg.edu.br \\ monilly.ramos@gmail.com, isabellemaria@copin.ufcg.edu.br
}

\begin{abstract}
Computational Thinking (CT) has grown over the last decade, although the identification and mapping of associated abilities is an open question. This paper presents a process that applies a statistical technique to guide the mapping of CT skills into questions. During the process, network analysis was applied in a dataset of 15 CT questions that do not require prior knowledge in programming or computing. The results pointed out that the items address more than one ability and can be grouped by the common abilities. Besides, the most explored ability in the set of questions was algorithmic thinking.
\end{abstract}

Resumo. A pesquisa em Pensamento Computacional $(P C)$ cresceu na última década, embora a identificação e mapeamento das habilidades associadas ainda seja uma questão em aberto. Este artigo apresenta um processo que emprega uma técnica estatística para nortear o mapeamento de habilidade de PC através de questões em uma prova. Durante o processo, análise de redes foi aplicada em um banco de respostas de 15 questões que demandam PC na resolução, mas que não necessitam de conhecimento prévio em programação. Os resultados apontaram que as questões endereçam mais de uma habilidade no processo de resolução e que podem ser agrupadas pelas habilidades em comum. Ademais, a habilidade mais explorada nas questões foi pensamento algorítmico.

\section{Introdução}

Pensamento Computacional (PC) é um tema de crescente interesse na área de Computação e Educação. A definição mais ampla afirma que PC constitui-se um processo de resolução de problemas explorando habilidades comuns da área de Ciência da Computação [Wing 2006, Bordini et al. 2017, Raabe et al. 2017]. O processo envolve empregar habilidades cognitivas, ou seja, construtos na resolução desses problemas. Construtos são variáveis que não podem ser medidas diretamente (como tempo ou velocidade), mas que precisam de um instrumento que permita a observação por meio do instrumento 
VII Congresso Brasileiro de Informática na Educação (CBIE 2018)

Anais do XXIX Simpósio Brasileiro de Informática na Educação (SBIE 2018)

[Hutz et al. 2015]. No nosso contexto, as habilidades do PC são construtos que precisam de instrumentos para serem observadas. Esses instrumentos podem ser testes, jogos, artefatos de código ou entrevistas.

Embora a pesquisa em PC concentre-se na Educação Básica, estudantes de graduação também podem se beneficiar, principalmente graduandos em Ciência da Computação e cursos afins [Bordini et al. 2017]. É notório que já se espera que estudantes de Computação desenvolvam habilidades do pensamento computacional, mas são poucas as evidências sobre como essas habilidades são desenvolvidas e os benefícios que pesquisas dessa natureza podem apontar. Por exemplo, explorar PC em estudantes de Computação pode auxiliar professores e pesquisadores a entender o relacionamento entre as habilidades do PC e as habilidades de programação em diferentes níveis (introdutório, intermediário e avançado).

Apesar do crescente interesse em PC, poucos estudos abordam sobre como identificar e mapear as habilidades do PC. Os estudos concentram-se em avaliar PC por meio de artefatos de código [Barcelos et al. 2017, Bordini et al. 2017, Araujo et al. 2016]. Por outro lado, os estudos que avaliam habilidades do PC sem o uso de programação são poucos [Raabe et al. 2017] ou possuem limitações para serem replicados [Araujo et al. 2016]. Além disso, a identificação de habilidades sem o uso de artefatos de código sofre da limitação de não poder ser totalmente automatizada e necessitar de análise de especialistas. Ainda assim, os trabalhos existentes nessa linha não utilizam análise estatística para apoiar o mapeamento de habilidades nas questões [Pessoa et al. 2017, Costa et al. 2016, Mestre et al. 2015].

Neste artigo, endereçamos a seguinte questão de pesquisa: Como mapear habilidades do PC em questões de maneira empírica guiado por um método estatístico? Assim, o objetivo deste trabalho é apresentar um processo de mapeamento de habilidades do PC em questões norteado por análise estatística de dados empíricos. Para atingir esse objetivo, selecionamos uma prova como instrumento contendo questões de PC de uma iniciativa internacional chamada Bebras ${ }^{1}$ e aplicamos em uma turma de ingressos no curso de Ciência da Computação. O instrumento contém 15 questões, todas relacionadas ao PC, mas sem requisitar conhecimento prévio de programação. Durante o processo, os dados coletados foram analisados empregando a técnica de análise de rede de correlações [Epskamp et al. 2012] no intuito de nortear o mapeamento de habilidade de PC nas questões do instrumento.

O restante do artigo está organizado da seguinte maneira: a Seção 2 apresenta conceitos sobre as habilidades do PC, a iniciativa internacional de estimular o PC chamada Bebras e os trabalhos relacionados. A Seção 3 descreve sucintamente a técnica de análise de rede. A Seção 4 detalha a metodologia adotada. As Seções 5 e 6 relatam e discutem os resultados, respectivamente. Por fim, a Seção 7 apresenta as considerações finais.

\section{Pensamento Computacional}

Nesta seção apresentamos habilidades relacionadas ao PC adotadas neste trabalho, a iniciativa internacional Bebras e os trabalhos relacionados ao tema do artigo.

\footnotetext{
${ }^{1}$ www.bebras.org
} 
VII Congresso Brasileiro de Informática na Educação (CBIE 2018)

Anais do XXIX Simpósio Brasileiro de Informática na Educação (SBIE 2018)

\subsection{Habilidades}

Computing At School ${ }^{2}$ é uma organização responsável por promover direcionamentos sobre o ensino de Computação no Reino Unido. Ela organizou um guia com conceitos e aplicações sobre PC para professores e pesquisadores da área [Csizmadia et al. 2015]. Assim, neste artigo, tomamos como base os conceitos das habilidades descritas pela Computing At School.

PC envolve raciocínio juntamente com um conjunto de habilidades no intuito de resolver problemas. Esse conjunto de habilidades inclui abstração, pensamento algorítmico, decomposição, generalização e avaliação [Csizmadia et al. 2015]. Abstração torna o problema mais fácil de ser compreendido reduzindo detalhes desnecessários e focando nas características essenciais. Abstração envolve também escolher representações mentais mais apropriadas que auxiliem a compreensão e a resolução do problemas. Já pensamento algorítmico é uma forma de organizar a solução em passos ordenados. Envolve também pensar em termos de sequência e regras como forma de encontrar a solução ou entender o problema.

Decomposição é uma estratégia de pensar em partes ou em componentes do problema. Essas partes podem ser tratadas e solucionadas separadamente para compor a solução completa do problema. Já generalização está associada a identificar padrões, similaridades e conexões para encontrar a solução do problema. Podemos identificar soluções de outros problemas similares e aplicá-las a novos problemas fazendo pequenas adaptações. Por último, avaliação é capacidade de identificar se uma solução proposta é adequada para o problema e se atende aos requisitos.

\subsection{Bebras}

Bebras é uma iniciativa internacional para estimular a disseminação do PC na educação básica, mas não limitada apenas às escolas. A primeira parte das atividades da equipe Bebras consiste em elaborar questões em um workshop anual envolvendo professores e pesquisadores de mais de 50 países [Dagienè et al. 2017]. As questões elaboradas ficam disponíveis em inglês e os países participantes selecionam de 15 a 18 questões para traduzir e compor as provas das suas competições locais. A segunda parte consiste nas competições locais, onde as provas são aplicadas online ou impressas nas escolas.

As questões da prova são elaboradas de forma que abordem um conteúdo de Computação e explorem habilidades do PC para resolvê-las. Entretanto, o estudante não precisa ter conhecimento prévio em Computação ou programação para resolver as questões, ou seja, as questões são autoexplicativas e autocontidas. Além disso, as questões Bebras podem explorar uma ou mais habilidades do PC na sua resolução [Dagienė et al. 2017]. As principais habilidades exploradas nas questões são abstração, pensamento algorítmico, decomposição, avaliação e generalização.

\subsection{Trabalhos Relacionados}

Barcelos et al. (2017) utilizaram código Scratch para obter inferências sobre o desenvolvimento de habilidades do PC de forma semi-automatizada. As habilidades mensuradas por meio de código foram abstração, lógica, controle de fluxo, representação de dados,

\footnotetext{
${ }^{2} \mathrm{https}: / /$ www.computingatschool.org.uk/
} 
VII Congresso Brasileiro de Informática na Educação (CBIE 2018)

Anais do XXIX Simpósio Brasileiro de Informática na Educação (SBIE 2018)

paralelismo, sincronização e interação com usuário. Essas habilidades estão fortemente relacionadas com a atividade de programar.

Ainda são poucos os estudos que buscam identificar habilidades do PC sem o uso de programação. Raabe et al. (2017) apresentaram um jogo puzzle capaz de identificar o desenvolvimento de habilidades do PC. O jogo aborda 20 habilidades e atitudes apresentadas pela Computer Science Teachers Association (CSTA).

Os estudos que empregam questões utilizam apenas especialistas para julgar a resolução das questões e identificar as habilidades presentes, sem apoio de métodos estatísticos. Pessoa et al. (2017) desenvolvam um aplicativo contendo questões que envolviam habilidades do PC na sua resolução. Por último, os trabalhos de [Costa et al. 2016] e de [Mestre et al. 2015] identificaram habilidades de PC em questões de matemática por meio de análise das respostas e discussão entre os especialistas. Todos os trabalhos relatados buscam, por diferentes vertentes (código, jogo e questões sem análise estatística), identificar habilidades do pensamento computacional.

\section{Análise de Rede}

No contexto de Ciência das Redes, uma rede pode ser compreendida como representação visual de dados empíricos por meio de modelos matemáticos os quais combinam algoritmos e técnicas gráficas [Hutz et al. 2015]. Uma rede representa um sistema de dados no qual as variáveis ou objetos são os nós, ou nodos, e as relações entre esses nós são representadas por linhas ou setas, chamadas de arestas. As redes possuem ampla aplicação na computação, física, ciências biológicas, ciências sociais e psicometria, pela vantagem de conseguir representar fenômenos complexos e identificar correlações entre variáveis.

Uma rede pode ter várias propriedades, dentre elas destacamos redes ponderadas e não ponderadas [Epskamp et al. 2012]. Nas redes não ponderadas, as linhas indicam apenas que há relação entre as variáveis, sem indicar intensidade. Já nas redes ponderadas, além de indicar as relações, as arestas indicam também a direção da associação (por meio de cores) e a intensidade dessas relações (por meio da espessura). Por padrão, a cor verde indica uma relação positiva e a cor vermelha, uma relação negativa [Epskamp et al. 2012]. Já as arestas de espessuras mais grossas indicam relações mais intensas ou fortes, enquanto que as arestas de espessura fina indicam relações fracas.

O posicionamento do nó na rede é outra propriedade que apresenta informação extra sobre as relações entre as variáveis. $\mathrm{O}$ algoritmo Fruchterman-Reingold posiciona os nós na rede da seguinte maneira [Epskamp et al. 2012]: inicialmente, todos os nós são repelidos. Então, os nós com maior intensidade de relação são atraídos entre si, formando agrupamentos, e os de baixa intensidade ou nula são repelidos. Os nós posicionados no centro da rede são aqueles que de certa maneira possuem mais relações intensas com os demais do sistema.

Para a psicometria, a análise de rede pode ser empregada para identificar construtos que emergem dos dados empíricos [Hutz et al. 2015]. Uma vez coletados dados de um instrumento e aplicada a análise de rede, podemos visualizar os agrupamentos dos itens do instrumento e discutir sobre qual construto pode explicar tais agrupamentos. Assim, a análise de rede é uma técnica emergente para identificação de construtos. 
VII Congresso Brasileiro de Informática na Educação (CBIE 2018)

Anais do XXIX Simpósio Brasileiro de Informática na Educação (SBIE 2018)

Tabela 1. Itens do instrumento

\begin{tabular}{|l|l|l|l|l|l|}
\hline Itens & Questões & Itens & Questões & Itens & Questões \\
\hline i1 & Cerimonia & i6 & Labirinto espacial & i11 & Ponto de encontro \\
\hline i2 & Artes em toras & i7 & Pegadas & i12 & Melhor tradução \\
\hline i3 & Castores em fuga & i8 & Saltos & i13 & Máquina quebrada \\
\hline i4 & Tráfego na vila & i9 & Rede social & i14 & Verdadeiro ou Falso \\
\hline i5 & Rede à prova de tempestade & i10 & Jogo da altura & i15 & Retângulos \\
\hline
\end{tabular}

\section{Metodologia}

Esta seção descreve o processo de mapeamento das habilidades do PC, o qual envolve as seguintes etapas: 1) aplicar e corrigir o instrumento; 2) realizar análise estatística; 3 ) identificar os agrupamentos de itens; 4) mapear as habilidades; e 5) validar o mapeamento.

Na Etapa 1, utilizamos como instrumento uma prova Bebras contendo 15 questões. A prova estava originalmente em inglês disponibilizada pela comunidade Bebras do Reino Unido em [Blokhuis et al. 2014]. As 15 questões selecionadas foram aquelas destinadas ao grupo de alunos de idade mais avançada (acima de 16 anos). Elas foram traduzidas e revisadas por dois revisores externos. Selecionamos essa prova porque o Brasil ainda não participou de competições Bebras e por isso, não existem questões em português. Apresentamos as questões enumeradas de i1 a i15 e seus respectivos nomes na Tabela 1. Os itens são do tipo múltipla escolha ou respostas curtas abertas. Independente do tipo, cada um é corrigido como certo ou errado, caracterizando que o instrumento possui itens dicotômicos. Os participantes deste estudo foram 100 estudantes ingressos no curso de Ciência da Computação regularmente matriculados em Programação I em 2017. Todos os estudantes das turmas foram convidados a participar e a seleção da amostra aconteceu por conveniência em adesão à pesquisa.

$\mathrm{Na}$ Etapa 2, a análise estatística procedeu pela aplicação da análise de rede por matriz de correlação realizada no RStudio por meio da biblioteca qgraph [Epskamp et al. 2012]. Os dados coletados foram submetidos à função qgraph configurando parâmetros de posicionamento e ponderação. Devido ao fato dos itens serem dicotômicos, utilizamos correlação tetracórica para produzir a rede.

Na Etapa 3, identificamos os agrupamentos dos itens. Prosseguimos para a Etapa 4, na qual realizamos o mapeamento das habilidades por agrupamento. Essa é uma atividade teórica que envolve identificar as habilidades que são exploradas para responder as questões. Por último, seguimos para Etapa 5, na qual o mapeamento de habilidades passa por uma validação por dois especialistas externos.

\section{Resultados}

Esta seção apresenta os resultados dos dados coletados. O instrumento aplicado e os dados estão disponíveis para replicação ${ }^{3}$. Na Etapa 1, identificamos a média de acertos no instrumento de 10.04 questões com desvio padrão de 2.26.

Na Etapa 2, a Figura 1 mostra o grafo resultante da correlação tetracórica de todos os 15 itens do instrumento. Podemos observar quatro tipos de relações. No primeiro tipo, os itens que mais se correlacionam estão representados por meio da aresta grossa na cor

\footnotetext{
${ }^{3}$ Omitido por ser blind review.
} 


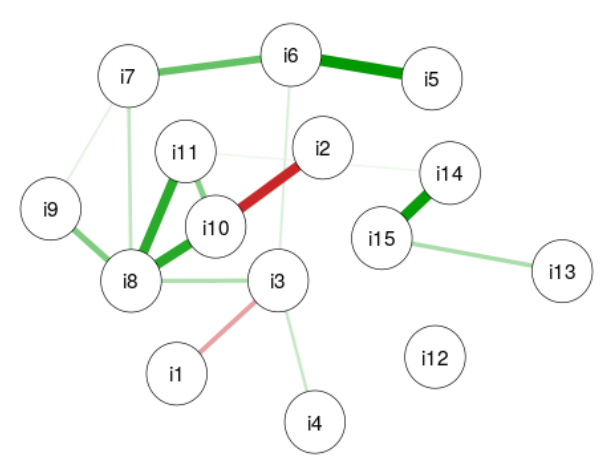

Figura 1. Análise de Rede considerando todos os 15 itens

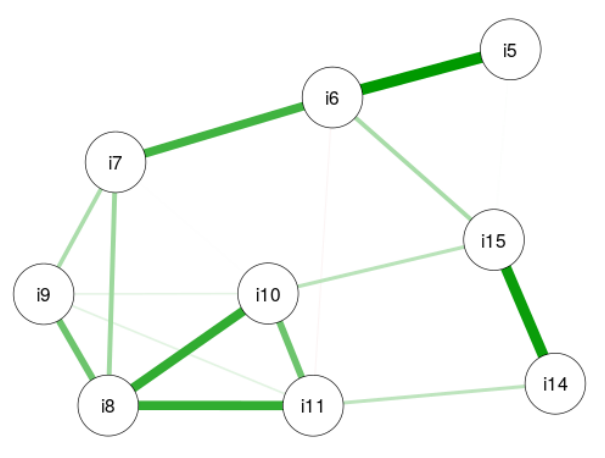

Figura 2. Análise de Rede dos itens selecionados

verde, formando três agrupamentos: itens 5,6 e 7; itens 8, 9, 10 e 11; e itens 14 e 15 . No segundo tipo, notamos que o item 12 não possui correlação expressiva com os demais itens e por isso, não possui arestas. No terceiro tipo, vemos que os itens 3, 4 e 13 possuem correções fracas representadas pelas arestas finas na cor verde claro. Por fim, os itens 1 e 2 possuem correlações negativas com outros itens do conjunto, representadas pelas arestas na cor vermelha.

Na Etapa 3, observamos que, dos quatro tipos de relações apresentados anteriormente, apenas o primeiro nos interessa para análise, pois são os itens que possuem padrões semelhantes de resposta. Os itens dos três últimos tipos serão desconsiderados da análise neste estudo. Assim, plotamos novamente o grafo com os itens 5, 6, 7, 8, 9, 10, 11, 14 e 15 para observar o comportamento dos dados com mais detalhes. Dessa forma, a Figura 2 apresenta o grafo resultante da correlação tetracórica dos itens mais correlacionados no instrumento. Nele, podemos perceber com mais clareza os três agrupamentos de correlações, entre os itens 5, 6 e 7; itens 8, 9, 10 e 11; e itens 14 e 15 .

Cada agrupamento de correlações identificados entre os itens representa um construto que pode explicar esse comportamento, ou seja, pode existir uma ou mais habilidades cognitivas que justificam os correlacionamentos. Portanto, na Etapa 4, como temos três agrupamentos, apresentamos a seguir o possível construto principal em comum, sua descrição e um exemplo de questão para cada agrupamento.

\subsection{Descrição e exemplo do construto 1}

Os itens 5, 6 e 7 apresentam em comum a habilidade de abstração na resolução do problema. Em todos os itens é necessário reconhecer os elementos principais para encontrar resolução dos problemas e escolher representações apropriadas para a questão. Entretanto, abstração não é a única habilidade para resolução das questões desse agrupamento. No item 6, que apresentamos na Figura 3, o aluno precisa escolher qual alternativa possui a sequência de passos que leva o robô ao destino. Porém, o algoritmo das alternativas está criptografado, obrigando o aluno a reconhecer quais códigos, ou seja, qual representação é a mais apropriada, e que contém a sequências de passos que leva o robô ao destino. Por outro lado, para responder o item 5, além de usar abstração, é necessário fazer avaliações em busca da melhor resposta. Já no item 7, é preciso seguir um algoritmo e construir a resposta correta a partir de passos menores (decomposição). Essas outras habilidades envolvidas justificam o posicionamento dos itens no grafo: em linha, sendo que o item 
VII Congresso Brasileiro de Informática na Educação (CBIE 2018)

Anais do XXIX Simpósio Brasileiro de Informática na Educação (SBIE 2018)

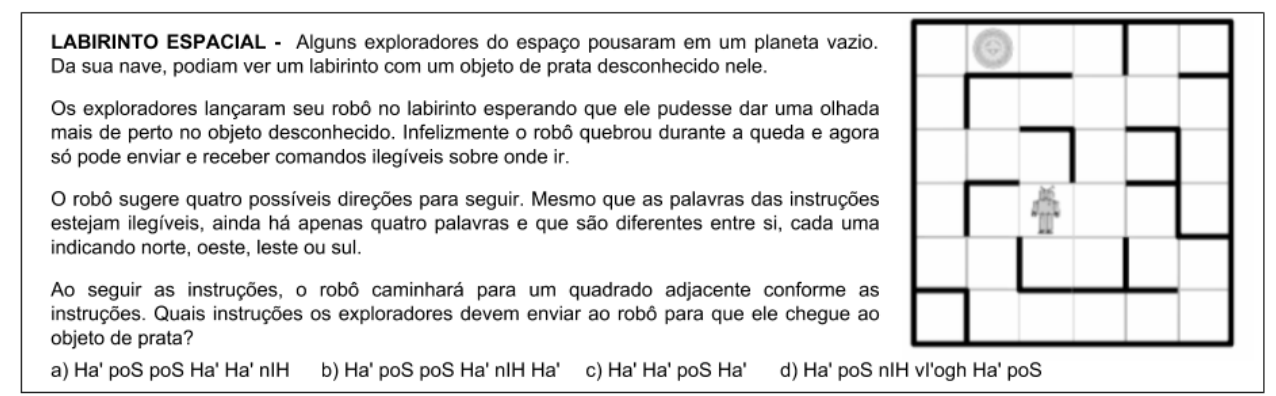

Figura 3. Labirinto Espacial (item 6)

6 é o central que faz a relação entre os itens 5 e 7, porque todos possuem em comum a habilidade de abstração para resolução dos três problemas.

\subsection{Descrição e exemplo do construto 2}

Os itens 8, 9, 10 e 11 apresentam em comum a habilidade de pensamento algorítmico e decomposição para resolução das questões. Os itens envolvem a atividade de decompor o problema em parte menores e seguir um conjunto de passos ou regras para compor a solução final. Apresentamos o item 8 na Figura 4 como exemplo. Nele, o aluno precisa executar o algoritmo descrito no enunciado, por etapas, para decidir o caminho de cada estudante. As habilidades de decomposição e pensamento algorítmico também são suficientes para resolver o item 10. Além de pensamento algorítmico e decomposição serem comuns a todas as questões, o item 9 explora ainda as habilidades de abstração e avaliação, enquanto que o item 11 , a habilidade de avaliação.

\subsection{Descrição e exemplo do construto 3}

Os itens 14 e 15 usam as habilidades de pensamento algorítmico e avaliação para responder ambas as questões. As questões apresentam passos ou regras que precisam ser seguidas, bem como avaliar a resposta que se adéqua à solução. $\mathrm{O}$ item 14 é apresentado na Figura 5. Nesse item, o aluno precisa avaliar as regras descritas em cada alternativa e executá-las na figura da questão para encontrar a resposta adequada. Ambos os itens $14 \mathrm{e}$ 15 compartilham das mesmas habilidades.

\subsection{Validação}

Na Etapa 5, dois especialistas da área de PC (um mestre e um doutorando) foram convidados a realizar o mapeamento das questões tendo como base o resultado da análise
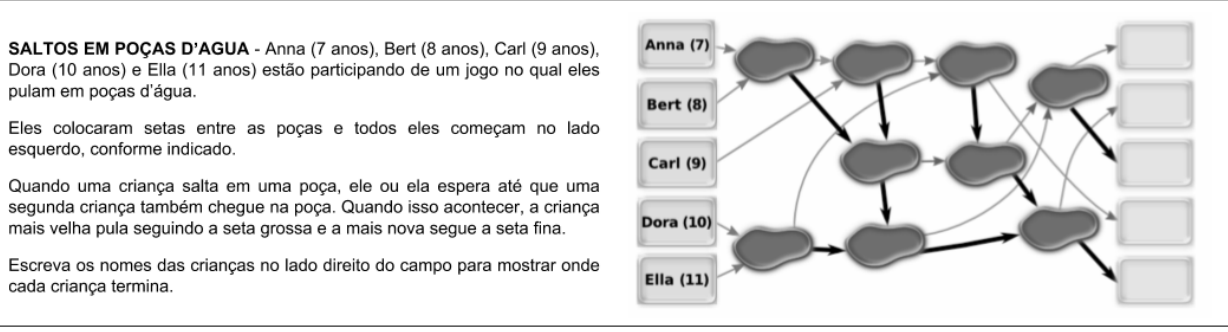

Figura 4. Saltos em Poças d’água (item 8) 
VII Congresso Brasileiro de Informática na Educação (CBIE 2018)

Anais do XXIX Simpósio Brasileiro de Informática na Educação (SBIE 2018)

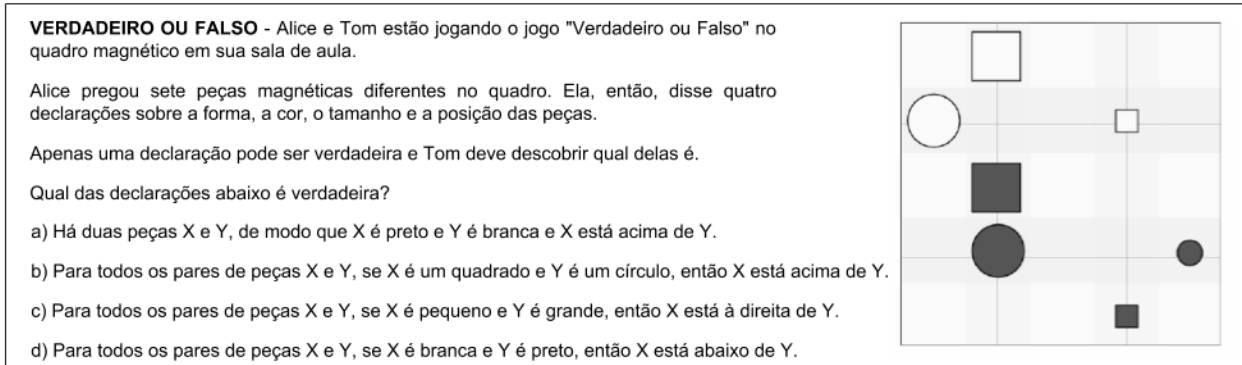

Figura 5. Verdadeiro ou Falso (item 14)

de redes. O resultado apontou concordância de ambos os especialistas aos construtos 1 e 3, embora mais habilidades tenham sido mapeadas. No construto 1, todos apontaram abstração como construto em comum. Em adição, o segundo especialista apontou que pensamento algoritmo também é habilidade presente nos itens 5, 6 e 7. Já no construto 3, além de pensamento algoritmo e avaliação apontados pelos dois especialistas, o segundo ainda apontou que abstração também é comum aos itens 14 e 15.

Em relação ao construto 2, o especialista 1 apontou que pensamento algoritmo e decomposição são as habilidades em comum, concordando com nossos resultados neste estudo. Entretanto, o segundo especialista apontou que pensamento algoritmo e abstração são as habilidades em comum para as questões 8, 9, 10 e 11. Para o item 8 (Figura 3), por exemplo, esse último especialista apontou que apenas pensamento algoritmo e abstração são as habilidades suficientes para resolver a questão.

A avaliação dos especialistas concordou em partes com o mapeamento realizado neste estudo. A divergência aconteceu em um dos especialistas que mapeou uma habilidade a mais para o construto 1 e não mapeou a habilidade de decomposição para o construto 2, sugerindo que abstração também faz parte desse construto. Ademais, todos eles concordaram com a presença de pensamento algorítmico em todas os itens, com exceção do item 5 para o primeiro especialista.

\section{Discussão}

Esta seção apresenta uma breve discussão sobre os resultados encontrados no processo no tocante às correlações entre os itens e às habilidades mapeadas como construtos.

As arestas finas na cor verde claro na Figura 1 são esperadas, pois acreditamos que todos os itens tratem de habilidades do PC. Dessa forma, é esperado que existam correlações fracas entre os itens, pois há um construto de mais alta granularidade (PC) sendo explorado pelos itens. Exemplos dessas correlações mais fracas são itens 6 e 15, itens 7 e 8 , itens 7 e 9, itens 11 e 14 .

Os itens dos agrupamentos apontados não compartilham uma única habilidade, como geralmente é esperado em instrumentos para avaliar construtos. Conseguimos caracterizar ao menos uma habilidade em comum por agrupamento, somada com outra(s) habilidade(s) complementar(es) particular(es) de cada item. Isso pode apontar a complexidade em elaborar questões que explorem apenas uma habilidade relacionada ao PC.

Todas as questões avaliadas, com exceção da 5, exploram a habilidade de pensamento algorítmico. A quantidade de questões que usam essa habilidade pode ser explicada 
VII Congresso Brasileiro de Informática na Educação (CBIE 2018)

Anais do XXIX Simpósio Brasileiro de Informática na Educação (SBIE 2018)

pelo fato de pensamento algorítmico ser apontada como uma das principais habilidades exploradas em estudos de PC, como também por ser considerada essencial no contexto de PC [Bordini et al. 2017, Araujo et al. 2016].

Observando que o item 8 (Figura 4) e o item 14 (Figura 5), estão em agrupamentos diferentes, podemos sugerir que pensamento algorítmico pode ser dividido em duas subhabilidades. A primeira delas é a mais comum: seguir passos pré-definidos para encontrar uma solução. Observamos esse processo no item 8 (Figura 4). A segunda sub-habilidade é pensar em termos de sequências e regras. Nesse caso, não há um único conjunto de passos a ser seguido, e sim regras que precisam ser consideradas para se chegar a solução. Exemplo dessa sub-habilidade está no item 14 (Figura 5). Entretanto, são necessários mais estudos teóricos e empíricos para poder afirmar essas sub-habilidades relacionadas a habilidade de pensamento algorítmico.

\subsection{Ameaças à Validade}

Este estudo possui ameaças à validade. O tamanho da amostra não permite generalizar os resultados. As questões foram corrigidas manualmente, e embora tenham passado por dupla checagem, podem acontecer erros de correção devido a fatores humanos. $\mathrm{O}$ instrumento utilizado foi traduzido do inglês, e embora cada item tenha sido revisado por dois revisores externos, pode conter viés de tradução por não representar totalmente a intenção original dos itens.

\section{Considerações Finais}

Este artigo apresentou um processo de mapeamento de habilidades do PC e questões. Utilizamos análise de rede com o intuito de empregar resultados estatísticos para nortear a associação das habilidades às questões. Assim, apresentamos uma proposta de mapear habilidades do PC em questões de maneira empírica guiada por um método estatístico. Como resultado, questões de um instrumento foram mapeadas nas habilidades de acordo com as correlações das respostas. As habilidades comuns nos três agrupamentos apontados pela análise de rede foram: (i) abstração, (ii) pensamento algorítmico e decomposição, e (iii) pensamento algorítmico e avaliação. Os resultados indicaram também que todos os itens exploram mais de uma habilidade na resolução. Além disso, pensamento algorítmico foi a habilidade mais comum identificada nesse conjunto de questões.

O mapeamento das habilidades que compõe cada item, e consequentemente cada agrupamento, é uma atividade teórica, ou seja, é inerente da interpretação dos dados empíricos frente aos conceitos das habilidades. Embora deseje-se automatizar o processo de mapeamento de habilidades em questões, ainda é necessária a análise humana no julgamento das habilidades empregadas durante a resolução. Assim, o mapeamento deste estudo foi validado por dois especialista. Entretanto, como as definições de cada habilidade do PC não são consenso dentro da área, isso é um dos fatores que dificulta existir uma classificação única. Ademais, uma questão pode ter processos distintos de resolução, que exploram habilidades diferentes. Por isso, o mapeamento apresentado neste estudo pode sofrer variações dependendo da interpretação das habilidades, bem como do processo de resolução das questões adotados.

A principal contribuição deste estudo é explorar uma técnica estatística para nortear o processo de mapeamento de habilidades em questões. Ademais, abordamos habilidade de PC como construtos e por isso, defendemos o uso de técnicas de mensuração 
VII Congresso Brasileiro de Informática na Educação (CBIE 2018)

Anais do XXIX Simpósio Brasileiro de Informática na Educação (SBIE 2018)

psicológica e interpretação cognitiva para auxiliar na identificação e mapeamento das habilidades. Análise de redes é um paradigma novo quando comparado com outras técnicas, mas já apresenta resultados validados na psicologia, e aponta resultados promissores também na área de PC quando abordamos as habilidades como construtos. Como trabalhos futuros, planejamos investigar outras formas de auxiliar a identificação e o mapeamento de habilidades do PC em questões apoiadas por resultados estatísticos.

\section{Referências}

Araujo, A. L. S. O., Andrade, W. L., and Guerrero, D. D. S. (2016). A systematic mapping study on assessing computational thinking abilities. In Frontiers in education conference (FIE), 2016 IEEE. IEEE.

Barcelos, T., Souza, A., Silva, L., Muñoz, R., and Acevedo, R. V. (2017). Mensurando o desenvolvimento do pensamento computacional por meio de mapas auto-organizáveis: um estudo preliminar em uma oficina de jogos digitais. In Anais dos VI Workshops do CBIE.

Blokhuis, D., Millican, P., Roffey, C., Schrijvers, E., and Sentance, S. (2014). UK Bebras Computational Thinking Challenge. Disponível em : http://www.bebras.uk/uploads/2/1/8/6/21861082/ukbebras2014-answers_1.pdf.

Bordini, A., Avila, C., Marques, M., Foss, L., and Cavalheiro, S. (2017). Pensamento computacional nos ensinos fundamental e médio: uma revisão sistemática. In XXVIII SBIE.

Costa, E. J. F., Sampaio, L., and Guerrero, D. (2016). Pensamento computacional na educação básica: Uma análise da relação de questões de matemática com as competências do pensamento computacional. In Anais dos V Workshops do CBIE.

Csizmadia, A., Curzon, P., Dorling, M., Humphreys, S., Ng, T., Selby, C., and Woollard, J. (2015). Computational thinking: A guide for teachers. Google Scholar.

Dagienè, V., Sentance, S., and Stupurienè, G. (2017). Developing a two-dimensional categorization system for educational tasks in informatics. Informatica, 28(1):23-44.

Epskamp, S., Cramer, A. O. J., Waldorp, L. J., Schmittmann, V. D., and Borsboom, D. (2012). qgraph: Network visualizations of relationships in psychometric data. Journal of Statistical Software, 48(4):1-18.

Hutz, C. S., Bandeira, D. R., and Trentini, C. M. (2015). Psicometria. Artmed Editora.

Mestre, P., Andrade, W., Guerrero, D., Sampaio, L., da Silva Rodrigues, R., and Costa, E. (2015). Pensamento computacional: Um estudo empírico sobre as questões de matemática do pisa. In Anais dos IV Workshops do CBIE.

Pessoa, F. I. R., Araujo, A. L. S. O., Andrade, W., and Guerrero, D. (2017). T-mind: um aplicativo gamificado para estímulo ao desenvolvimento de habilidades do pensamento computacional. In XXVIII SBIE.

Raabe, A., Santana, A. L. M., Ellery, N., and Gonçalves, F. (2017). Um instrumento para diagnóstico do pensamento computacional. In Anais dos VI Workshops do CBIE.

Wing, J. M. (2006). Computational thinking. Communications of the ACM, 49(3):33-35. 